\title{
Les Pathologies Chirurgicales Néonatales À l'Hôpital National De Lamordé De Niamey: Aspects Diagnostiques, Thérapeutiques Et Pronostiques
}

\section{Ali Ada Mahamoud Omid (Docteur MD.) Moustapha Hellé (Docteur MD.)}

Faculté des Sciences de la Santé, Université Abdou Moumouni de Niamey, Hôpital National de Lamordé, Niger

Habou Oumarou (Docteur MD.)

Faculté des Sciences de la Santé, Université de Zinder, Hôpital National de Zinder, Niger

Cherif Maiguizo Abdoul Karim (Docteur MD.)

Hôpital National de Lamordé, Niger

Abarchi Habibou (Professeur, Docteur MD.)

Faculté des Sciences de la Santé, Université Abdou Moumouni de Niamey, Hôpital National de Lamordé, Niger

doi: 10.19044/esj.2017.v13n24p156 URL:http://dx.doi.org/10.19044/esj.2017.v13n24p156

Abstract

Purpose: The purpose of this study is to describe the characteristics of the newborn and its surgical pathologies managed in the service of pediatric surgery of the National Hospital of Lamordé. Patients and Methods: It is a retrospective and descriptive study within a period of 10 years (from January 1st 2007 till December 31st 2016). The epidemiological, diagnostic, therapeutic, and evolutionary aspects were also studied. Results: The surgical pathologies of the newborn child represented $12.8 \%$ of the hospitalized patients. The average annual incidence was 47.5 newborn children a year. The average age was of 8.71 days. The newborn children were especially old from 0 to 7 days $(60,2 \%)$ and were male $(66.1 \%)$. The most frequent pathologies were the malformations anorectales $(32,2 \%)$, the omphalocele $(18.3 \%)$, and the gastrochisis $(6.5 \%)$. The surgical operations, realized for $67.7 \%$ patients, were especially emergencies $(58.2 \%)$. The colostomy was the most realized intervention $(41.9 \%)$. The mortality rate was $25.8 \%(\mathrm{n}=123)$. The post-operative mortality was $16.9 \%$ and the nonoperative mortality was $45 \%$. The premature mortality established $70 \%$ of deaths $(\mathrm{n}=75)$. The omphalocele was the main pathology supplier of death $(26 \%)$. Conclusion: The neonatal surgical pathologies are characterized by 
their frequency, their diversity, the predominance of digestive system pathologies, and a high mortality. An evaluation of pronostic factors which determines the morbi-mortality is necessary.

Keywords: Surgery, neonatal, newborn, Niger

\section{Résumé}

But: Le but de ce travail était décrire les caractéristiques du nouveau-né et ses pathologies chirurgicales prises en charge au service de chirurgie pédiatrique de l'Hôpital National de Lamordé.

Patients et Méthodes: Il s'agit d'une étude rétrospective et descriptive portant sur une période de 10 ans (du $1^{\mathrm{er}}$ janvier 2007 au 31 décembre 2016). Les aspects épidémiologiques, diagnostiques, thérapeutiques et évolutifs ont été étudiés. Résultats: Les pathologies chirurgicales du nouveau-né représentaient $12,8 \%$ des patients hospitalisés. L'incidence annuelle était de 47,5 nouveau-nés par an. La moyenne d'âge était de 8,71 jours. Les nouveau-nés étaient surtout âgés de 0 à 7 jours $(60,2 \%)$ et de sexe masculin $(66,1 \%)$. Les pathologies les plus fréquentes étaient les malformations anorectales $(32,2 \%)$, l'omphalocèle $(18,3 \%)$ et le laparoschisis $(6,5 \%)$. Les interventions chirurgicales, réalisées chez $67,7 \%$ des patients, étaient surtout des urgences $(58,2 \%)$. La colostomie était le geste le plus souvent réalisé (41,9\%). Le taux de mortalité était de $25,8 \% \quad(n=123)$. La mortalité postopératoire était de $16,9 \%$ et celle non opératoire de était de $45 \%$. La mortalité précoce constituait $70 \%$ des décès $(n=75)$. L'omphalocèle était la pathologie la plus pourvoyeuse de décès (26\%). Conclusion: Les pathologies chirurgicales néonatales sont caractérisées par leur fréquence, leur diversité, la prédominance des pathologies de l'appareil digestif et une mortalité précoce et élevée. Une évaluation des facteurs pronostiques déterminant la morbi-mortalité est nécessaire.

Mots-clés: chirurgie, néonatale, nouveau-né, Niger

\section{Introduction}

La chirurgie néonatale correspond aux soins chirurgicaux destinés aux enfants dont l'âge est inférieur ou égale à 28 jours (Arul, 1998). Elle est l'une des composantes les plus difficiles et la sous spécialité la plus jeune de la chirurgie pédiatrique. La période néonatale qui correspond aussi à la période d'adaptation à la vie extra-utérine se distingue des autres classes d'âge par de plus grandes spécificités anatomiques et physiologiques. Les pathologies rencontrées sont essentiellement malformatives. Il s'agit le plus souvent d'urgence chirurgicale mettant en jeu le pronostic vital et/ou fonctionnel. La chirurgie néonatale est pratiquée dans les centres spécialisés. 
La prise en charge est lourde et nécessite un personnel qualifié et du matériel spécifique. Elle représenterait environ $10 \%$ des activités chirurgicales au sein des différents services de chirurgie pédiatrique en Afrique (Chirdan, 2012). D'énormes progrès ont été réalisés durant les dernières décennies avec pour résultat une diminution significative de la mortalité chirurgicale néonatale. Cependant ce taux de mortalité reste encore très élevé dans les pays en voie de développement. Ceci est lié à l'insuffisance des ressources humaines et matérielles et à l'absence de politique de santé visant à réduire la mortalité néonatale chirurgicale (Arul, 1998; Chirdan, 2012).

En Afrique la chirurgie néonatale a fait l'objet de peu de travaux. Au Niger, il existe peu d'études concernant le spectre des pathologies chirurgicales néonatales (Harouna, 1997). Cette étude se propose ainsi de décrire le nouveau-né et ses pathologies pris en charge au sein du Service de Chirurgie pédiatrique de l'Hôpital National de Lamordé.

\section{Patients et Méthode}

Il s'agissait d'une étude rétrospective et descriptive réalisée au Service de Chirurgie pédiatrique de l'Hôpital National de Lamordé. Elle portait sur les nouveau-nés pris en charge dans le service durant une période de 10 ans allant du $1^{\text {er }}$ janvier 2007 au 31 décembre 2016. N'étaient pas inclus dans notre étude: les patients ayant été traités en chirurgie ambulatoire et les nouveau-nés vus en consultation externe. Les variables socio-démographiques (âge, sexe), cliniques, thérapeutiques et pronostiques ont été étudiées.

\section{Résultats}

Durant la période d'étude, 475 nouveau-nés ont été recensés. Ils constituaient $12,8 \%$ des 3708 patients pris en charge, soit une fréquence de 47,5 nouveau-nés par an. Cette fréquence annuelle est passée de 19 cas en 2007 à 89 cas en 2016.

La moyenne d'âge était de 8,71 jours (extrêmes : 0 et 28 jours) et $60,2 \%$ des patients étaient âgés de moins d'une semaine de vie ( $\mathrm{n}=286$ cas). Il y avait une prédominance du sexe masculin de $66,1 \%(n=314)$.

Les malformations anorectales étaient la pathologie la plus fréquente avec 153 cas $(32,2 \%)$ suivi de l'omphalocèle et du laparoschisis avec respectivement 87 cas $(18,3 \%)$ et 31 cas soit $6,5 \%$ (Tableau I).

$\mathrm{Au}$ total les malformations de l'appareil digestif étaient les plus fréquentes avec 325 cas soit $68,4 \%$, suivies des péritonites avec 24 cas (5\%).

Près de trois quarts des nouveau-nés admis avaient bénéficié d'une intervention chirurgicale : 322 patients soit $67,7 \%$. Les pathologies les plus fréquentes pour lesquelles les nouveau-nés étaient opérés étaient les 
malformations anorectales avec 148 cas $(31,1 \%)$, les péritonites (22 cas soit $4,6 \%$ ), et le laparoschisis (19 cas soit $4 \%$ ).

Les interventions chirurgicales ont été réalisées en urgence dans 58,2\% des cas. La colostomie était le geste le plus souvent réalisé avec 135 cas soit $41,9 \%$ des interventions chirurgicales réalisées (Tableau II).

Tableau I. Répartition des nouveau-né selon le diagnostic

\begin{tabular}{ccc}
\hline Diagnostic & Effectif & Pourcentage \\
\hline Malformation anorectale & 153 & 32,2 \\
Omphalocèle & 87 & 18,3 \\
Laparoschisis & 31 & 6,5 \\
Péritonite & 24 & 5 \\
Maladie de Hirschsprüng & 21 & 4,4 \\
Atrésie de l'œsophage & 19 & 4,1 \\
Cryptorchidie & 17 & 3,6 \\
Exstrophie vésicale & 16 & 3,3 \\
Hygroma kystique cervical & 16 & 3,3 \\
Fracture du fémur & 11 & 2,5 \\
Sténose hypertrophique du pylore & 10 & 2,1 \\
Abcès parties molles & 10 & 2,1 \\
Atrésie du côlon & 9 & 1,8 \\
Tumeur sacro-coccygienne & 9 & 1,8 \\
Atrésie du grêle & 8 & 1,7 \\
Syndrome de Prune Belly, anus, face) & 7 & 1,4 \\
Hernie inguinale ou inguino-scrotale & & \\
Jumeaux Siamois & 7 & 1,4 \\
Ambiguïté sexuelle & 5 & 1,1 \\
Maladie des brides amniotiques & 5 & 1,1 \\
Entérocolite ulcéronécrosante & 3 & 0,7 \\
Fasciite nécrosante & 3 & 0,7 \\
Hernie ombilicale étranglée & 1 & 0,225 \\
Sténose duodénale & 1 & 0,225 \\
Total & 1 & 0,225 \\
tumeur (abdomen, cou, thorax, & 1 & 0,225 \\
(1) & 475 & 100 \\
\hline & &
\end{tabular}

La durée moyenne d'hospitalisation était de 13,5 jours . La majorité des nouveaux nés $(62,3 \%)$ avait une durée d'hospitalisation supérieure à 1 semaine.

La mortalité globale était de $25,8 \%(\mathrm{n}=123)$. La mortalité précoce (décès survenant au cours de la première semaine de vie) constituait $70 \%$ des décès ( $\mathrm{n}=75$ cas). Pour les patients ayant subi une intervention chirurgicale , il a été noté 54 cas de décès soit un taux de mortalité post-opératoire de $16,7 \%$. Pour les patients non opérés $(n=153)$, le taux de mortalité était de $45 \%(n=69)$. L'omphalocèle, le laparoschisis et les malformations anorectales étaient les pathologies les plus pourvoyeuses de décès avec respectivement $26 \%(\mathrm{n}=32), 20,3 \%(\mathrm{n}=25)$ et $16,2 \%(\mathrm{n}=20)$. 
Tableau III. Répartition des patients selon le geste chirurgical

\begin{tabular}{ccc}
\hline Indication chirurgicale & Fréquence & Pourcentage \\
\hline Colostomie & 135 & $41,9 \%$ \\
Fermeture de la paroi abdominale & 27 & $8,3 \%$ \\
Exerese de masse & 23 & $7,1 \%$ \\
Fistulorraphie+rectoanoplastie & 18 & $5,5 \%$ \\
Fermeture plaque vésicale & 16 & $5,1 \%$ \\
Iléostomie & 14 & $4,3 \%$ \\
Résection-anastomose intestinale & 12 & $3,8 \%$ \\
Pylorotomie & 10 & $3,2 \%$ \\
Drainage d'abcès & 10 & $3,2 \%$ \\
Autres* & 57 & $17,6 \%$ \\
Total & 322 & $100 \%$ \\
\hline
\end{tabular}

* Les autres gestes étaient : appendicectomie, biopsie rectale, fixation de la grande courbure, jejunostomie, jejuno-duodenostomie, œsophagoplastie, orchidopexie, parage (fasciite nécrosante), posthectomie, promontofixation, pyélolithotomie, transposition anale, urétroplastie, Cure de hernie inguinale, Laparotomie exploratrice (ambiguïté sexuelle), Suture de perforation intestinale, Libération des brides, Cheiloplastie, Gastrostomie,

Hémicolectomie.

\section{Discussion}

Les pathologies chirurgicales néonatales représentaient 12,8\% des admissions dans notre service avec une incidence annuelle de 47,5 nouveaunés. Dans des séries africaines les pathologies chirurgicales néonatales représentaient 9,7\% à 61\% des admissions (Mieret, 2014; Doumbouya, 1999; Badrinath, 2014). Des études réalisées en Afrique (Osifo, 2008; Sowande 2007; Ndour, 2009; Keita, 2006) retrouvaient des incidences annuelles inférieures à la nôtre variant de 11 à 25 nouveau-nés par an. Dans des séries indiennes et britannique cette incidence était supérieure à la nôtre, 60 à 301 nouveau-nés par an (Narasimhan, 2005; Burge 2012; Nandi, 2008; Bhatnagar, 2012). Les caractéristiques démographiques des populations et les spécificités des services de chirurgie pédiatrique qui les recouvrent sont variables et déterminent l'incidence hospitalière.

La moyenne d'âge dans notre étude était de 8,71 jours. Dans des séries africaines et indiennes ont retrouve des moyennes d'âge inférieures à la nôtre allant de 5 à 8 jours (Badrinath, 2014; Osifo, 2008; Sowande 2007; Peyvasteha 2011; Ekenze, 2016; Gangopadyay, 2008; Osifo, 2009). La majorité des nouveau-nés était âgé de 0 à 7 jours à l'admission dans notre étude $(60,2 \%)$. Dans les études de Badrinath et col. en Ouganda, Osifo et col. au Nigeria les patients étaient aussi majoritairement admis au cours de la première semaine de vie avec respectivement $63,5 \%$ et $47,5 \%$ des cas. La gravité des tableaux cliniques qui se manifestent souvent dès la naissance 
motiverait les parents à faire consulter leur enfant tôt et en urgence (Badrinath, 2014; Osifo, 2008).

Dans notre série tout comme dans la littérature on note une prédominance masculine : respectivement $66,1 \%$ de garçons et entre $56 \%$ et $75 \%$ de nouveau-nés de sexe masculin (Badrinath, 2014; Osifo, 2008; Sowande 2007; Ndour, 2009; Narasimhan, 2005; Catré, 2013; Awad, 2014).

Le diagnostic le plus fréquent dans notre étude était les malformations anorectales, 32,2\% $(n=153)$, suivis des défects de la paroi abdominale antérieure : omphalocèle, $18,3 \%(\mathrm{n}=87)$ et laparoschisis, 6,5\% $(n=31)$. Dans d'autres études africaines (Chirdan, 2012; Badrinath, 2014; Sowande, 2007; Nandi, 2008; Ameh, 2001), les malformations anorectales étaient la pathologie la plus fréquemment retrouvée $\div$ variant entre $9,5 \%$ et $39 \%$ des cas. Dans une méta analyse portant sur la chirurgie néonatale en Afrique (51 études) les atrésies intestinales était observées dans 54,9\% des travaux, les défects de la paroi abdominale antérieure dans 52,9\% études et les malformations anorectales pour $47,1 \%$ des séries (Ekenze, 2016). Le spectre des admissions chirurgicales néonatales peut varier d'un hôpital à l'autre en fonction de la disponibilité des moyens de diagnostic prénatal, des facilités de transport et du délai de présentation.

Les malformations de l'appareil digestif ont constitué les cas les plus fréquents dans notre série, 68,4\% $(n=325)$. Elles prédominaient aussi dans d'autres études : entre 41,8\% et 82\% (Mieret, 2014; Osifo, 2008; Sowande, 2007; Awad 2014; Ugwu 2013).

Les nouveau-nés dans notre série étaient plus fréquemment opérés (67,7\%) que dans d'autres études : de 28,3\% à 52,7\% de nouveau-nés opérés (Mieret, 2014; Badrinath, 2014; Ugwu, 2013). Des variations dans les proportions entre les pathologies imposant une intervention chirurgicale en urgence (colostomie pour malformation anaorectale par exemple) et celles pour qui un traitement conservateur est possible (comme l'omphalocèle) pourrait expliquer une telle différence.

La mortalité est dans la plupart des études inférieure à la nôtre (25,8\%) variant de 3\% à 72,8\% (Sowande, 2007; Ndour, 2009; Peyvasteha, 2011; Osifo, 2009; Awad, 2014; Taguchi, 2014). Des avancées dans les techniques diagnostiques et les soins péri-opératoires ont permis d'améliorer grandement les résultats thérapeutiques de la chirurgie néonatale. Il existe malgré tout, des disparités en terme de résultats de la chirurgie néonatale entre les pays avec hauts revenus et ceux avec un revenu moyen et un revenu bas (Ekenze, 2016). Selon une méta-analyse ceci est dû à un retard de présentation et des installations inadéquates dans 39 études (76,5\%), le manque de personnel de soutien entrainé dans 32 séries $(62,7 \%)$ et l'absence de soins intensifs néonataux dans 29 études (56,9\%) (Ekenze, 2016). 
Les facteurs de morbidités et de mortalité identifiés dans différentes études étaient : les accouchements à domicile ou dans les structures de santé rurales, la prématurité et le faible poids de naissance, l'atteinte de l'appareil digestif, le transport non médicalisé, les problèmes nutritionnels, les complications anesthésiques et la présence d'anomalies congénitales multiples (Osifo, 2008; Nandi, 2008; Ugwa, 2013).

La mortalité post-opératoire dans notre étude était de 16,7\% (54 cas). Dans les pays en voie de développement la mortalité post-opératoire reste encore très élevée variant de 12,8\% à 62,3\% (Mieret, 2014; Badrinath, 2014; Osifo, 2008; Ndour, 2009).Cette variation de la mortalité pourrait être liée entre autre à la taille des échantillons et le type de structure où l'étude a été réalisée.

La mortalité non opératoire chez nos patients est plus élevée 45\% (69 cas) que la mortalité opératoire et elle constitue la majorité des décès (56\%). Ces résultats pourraient témoigner d'une part de la sévérité des tableaux cliniques à l'admission et d'autre part de l'insuffisance de moyens de prise en charge médico-chirurgicale adéquats. Dans la littérature une différence statistiquement significative entre la mortalité opératoire et la mortalité non opératoire n'est pas constamment observée. Elle est tantôt absente (Ugwu, 2013), tantôt présente avec une baisse de près de $12,8 \%$ en faveur des patients ayant bénéficiés d'une intervention chirurgicale (Badrinath, 2014). La mortalité chez nos patients était surtout une mortalité précoce : $70 \%$ des décès ( 75 cas). Badrinath et N'dour ont retrouvé cette prédominance de décès au cours de la première semaine avec respectivement $43,5 \%$ et $68 \%$ des cas (Badrinath, 2014; Ndour, 2009).

Les décès survenaient en premier lieu sur des cas d'omphalocèle ( $26 \%$ des décès néonataux) dans notre série. Mieret en Côte-d'Ivoire a retrouvé également une prédominance des omphalocèles pour les décès (39 cas soit 34,21\%) (Mieret, 2014). Pour d'autres auteurs il s'agissait plutôt des malformations ano-rectales (28\%) (Ralahy, 2010) ou des atrésies intestinales (35,9\%) (Osifo, 2009).

\section{Conclusion}

Les pathologies chirurgicales néonatales sont diverses et leurs incidences croissantes dans notre service. Elles sont majoritairement constituées de pathologies malformatives de l'appareil digestif constituant une urgence. La mortalité est très élevée et précoce. Elle est probablement liée au retard diagnostique, à l'insuffisance du plateau technique, l'absence d'unité réanimation néonatale. Des recherches appropriées, une approche multidisciplinaire, des investissements financiers adéquats et une meilleure sensibilisation de la population sont des actions nécessaires pour améliorer 
les soins chirurgicaux néonataux en Afrique et particulièrement dans notre pays.

\section{References:}

1. Ameh E. A, Dogo P. M, Nmadu P. T. (2001). Emergency neonatal surgery in developing country. Pediatr Surg Int ; 17 : 448-451.

2. Arul G. S, Spicer R. D. (1998). Where Should paediatric Surgery be performed ; Arch Dis Child, 79 : 65-70.

3. Awad R. A, Karasani S. H. (2014). Pattern of Neonatal Surgical Presentation and Outcome in Sinnar Hospital (2013-2014). Global Journal of Medical Research; 14 : 17-22.

4. Badawi N, Adelson P, Roberts C. (2003). Neonatal surgery in New South Wale - What is performed where? J Pediatr Surg; 38: 10251031.

5. Badrinath R, Kakembo N, Kissa P, Langer M, Ozgediz D, Sekabira J. (2014). Outcomes and unmet need for neonatal surgery in a ressouce limited environment: Estimates of global health disparities from Kampala Ouganda. J Ped Surg ; 49 : 1825-1830.

6. Bhatnagar S. N, Sarin Y. K. (2012). Current trends in neonatal surgery in India. J Neonat Surg ; 1 : 18-21.

7. Burge D. M, Drewett M. (2012). Workload and costs associated with providing a neonatal surgery service. Arch Dis Child Fetal Neonatal ; 97 : 179-181.

8. Catré D, Lopes M. F, Madrigal A, Oliveiro B, Viana J. S, Cabrita A. S. (2013). Early mortality after neonatal surgery analysis of risk factors in a optimized health care system for the surgical newborn. Rev Bras Epidemiol ; 16 : 945-52.

9. Chirdan L. B, Ngiloi P. J, Elhaby E.A. (2012). Neonatal surgery in Africa. Semin Pediatr Surg ; 21 : 151 - 159

10. Doumbouya N, Keita M, Magasouba D et al. (1999). Mortalité dans le service de chirurgie pédiatrique au CHU Donka. Med Afr Noire ; $46: 589-592$.

11. Ekenze S. O, Ajuzieogu O. V, Nwomeh B. C. (2016). Challenges of management and outcome of neonatal surgery in Africa : a systematic review. Pediatr Surg Int, 32 : 291-299.

12. Gangopadyay A. N, Upadyaya V. D, Sharma S. P. (2008). Neonatal Surgery: A ten year audit from university hospital. Indian J Pediatr ; 75 :1025-1030.

13. Harouna Y, Tardivel G, Bia M, Abdou I, Gamatie Y. (1997). Occlusion intestinale néonatale : Notre expérience à propos de 10 cas. Med Afr Noire ; 44 (12) : 648- 51. 
14. Keita M, Diallo M. S. A, Keita A. K, Diallo A. F, Balde I. (2006). Les urgences chirurgicales néonatales dans le service de chirurgie pédiatrique du CHU Donka. Mali Médical ; 21: 16-21.

15. Livingston M. H, Cruz J., Pemberton J, Ozgediz D., Poenaru D. (2015). Mortality of pediatric surgical conditions in low and middle income countries in Africa.. J Pediatr Surg ; 50 : 760-764.

16. Mieret J. C, Yaokreh J-B, Dieth A. G et al. (2014). Pronostic des pathologies chirurgicales néonatales au CHU de Yopougon. Rev Int Sc Méd; 16 : 126-129.

17. Narasimhan K. L, Vivay B. (2005). Priorities in development of neonatal surgery in India. Indian pediatrics; $42: 82-83$.

18. Nandi B, Mungougo C, Lakhoo K. (2008). A comparison of neonatal surgical admission between two linked surgical departments in Africa and Europa. Pediatr Surg Int ; 24 : 939-942.

19. Ndour O, Fall F.A, Alumeti D et al. (2009). Facteurs de mortalité néonatale dans le service de chirurgie pédiatrique du CHU Aristide Le Dantec de Dakar. Mali médical ; 14 : 33-38.

20. Osifo O. D., Ovueni M. E. (2009). The prevalence, patterns, and causes of deaths of surgical neonates at two African Referral Pediatric Surgical Centers. Ann Pediatr Surg ; 5 : 194-199.

21. Osifo O. D, Oriaifo I. A. (2008). Factors affecting the management and outcome of neonatal surgery in Benin city, Nigeria. Eur J Pediatr Surg ; 18 : 107-110.

22. Peyvasteha M, Askarpoura S, Javaherizadehb H, Fatahiana T. (2011). Evaluation of epidemiologic indices of neonate's diseases in the Pediatric Surgery Ward of the Ahvaz Jundishapur University hospitals during the period 1993-1996 and 2002-2005. Ann Pediatr Surg ; 7 : 7-9.

23. Ralahy MF, Rakotoarivoni ST, Rakotovao MA, Hunaald FA, Rabenasolo M, Andriamanarivo ML. (2010). La mortalité néonatale au service des urgences du CHUA-JRA Antananarivo-Madagascar. Rev Anesth Rea Med Urg ; 2 (Arul, 1998):15-17.

24. Sowande O. A, Ogundoyin O. O, Adejuyigbe O. (2007). Pattern and factors affecting management outcome of neonatal emergency surgery in Ile-lfe, Nigeria. Surg Pract ; 11 : 71-75.

25. Taguchi T, Nagata K, Kinoshita Y, Esumi G. (2014). Progress in and outcomes of neonatal surgery over the past 50 years. Nihon Geka Gakkai Zasshi.; 115 : 306-311.

26. Ugwu R. O, Okoro P. E. (2013). Pattern outcome and challenges of neonatal surgical cases in a tertiary teaching hospital. Afr J Pediatr Surg ; 10 : 226-230. 